\title{
Observational Evidence for Gentle Chromospheric Evaporation During the Impulsive Phase of a Solar Flare
}

\author{
Ryan O. Milligan ${ }^{1,3}$, Peter T. Gallagher ${ }^{2,3,4}$, Mihalis Mathioudakis ${ }^{1}$, and Francis P. Keenan ${ }^{1}$
}

\begin{abstract}
Observational evidence for gentle chromospheric evaporation during the impulsive phase of a C9.1 solar flare is presented using data from the Reuven Ramaty High-Energy Solar Spectroscopic Imager and the Coronal Diagnostic Spectrometer on board the Solar and Heliospheric Observatory. Until now, evidence for gentle evaporation has often been reported during the decay phase of solar flares, where thermal conduction is thought to be the driving mechanism. Here we show that the chromospheric response to a low flux of nonthermal electrons $\left(\geq 5 \times 10^{9} \mathrm{ergs} \mathrm{cm}^{-2} \mathrm{~s}^{-1}\right)$ results in plasma upflows of $13 \pm 16,16 \pm 18$, and $110 \pm 58 \mathrm{~km} \mathrm{~s}^{-1}$ in the cool He I and $\mathrm{O} V$ emission lines and the 8 MK Fe XIX line. These findings, in conjunction with other recently reported work, now confirm that the dynamic response of the solar atmosphere is sensitively dependent on the flux of incident electrons.
\end{abstract}

Subject headings: Sun: atmospheric motions - Sun: flares - Sun: UV radiation - Sun: X-rays, $\gamma$ rays

\section{INTRODUCTION}

During the impulsive phase of a solar flare, accelerated electrons propogate along closed magnetic field lines to the dense, underlying chromosphere, where they lose their energy via Coloumb collisions and heat the local plasma. The resulting expansion of this plasma is known as "chromospheric evaporation". From the hydrodynamic simulations of Fisher, Canfield, \& McClymont (1985a,b,c), and more recently Abbett \& Hawley (1999), the solar atmosphere is predicted to respond in one of two ways, depending on the flux of accelerated nonthermal electrons.

For electron fluxes $\leq 10^{10} \mathrm{ergs}_{\mathrm{cm}}^{-2} \mathrm{~s}^{-1}$, the evaporated plasma flows upwards at several tens of kilometers per second, with no associated downflows. This process is termed 'gentle' evaporation. Gentle evaporation may also occur during the de-

\footnotetext{
${ }^{1}$ Department of Physics and Astronomy, Queen's University Belfast, Belfast, BT7 1NN, Northern Ireland.

${ }^{2}$ School of Physics, Trinity College Dublin, Dublin 2, Ireland.

${ }^{3}$ Laboratory for Solar and Space Physics, NASA Goddard Space Flight Center, Greenbelt, MD 20771, U.S.A.

${ }^{4}$ L-3 Communications GSI.
}

cay phase, when the upflows are driven by thermal conduction rather than electron beam heating (Antiochos \& Sturrock 1978). Many studies have reported evidence for conduction driven evaporation during the decay phase of solar flares (Schmieder et al. 1987; Zarro \& Lemen 1988; Czaykowska et al. 2001; Berlicki et al. 2005). In each of these studies it was concluded that the late phase evaporation was caused by heat conduction along field lines connecting the chromosphere to the corona. Evidence for gentle evaporation was also presented by Brosius \& Phillips (2004) during flare precursor events, but the mechanism responsible could not be verified. To date, there has been no direct evidence for gentle evaporation due to nonthermal electrons during the impulsive phase of a solar flare.

At high nonthermal electron fluxes $\left(\gtrsim 3 \times 10^{10} \mathrm{ergs} \mathrm{cm}^{-2} \mathrm{~s}^{-1}\right)$, the chromosphere is unable to radiate at a sufficient rate and consequently expands rapidly. The heated chromospheric plasma $\left(\sim 10^{7} \mathrm{~K}\right)$ expands upwards at hundreds of kilometers per second in a process known as 'explosive' evaporation. The overpressure of the flare plasma relative to the underlying chromosphere also causes cooler, more dense material to expand downwards at tens of 

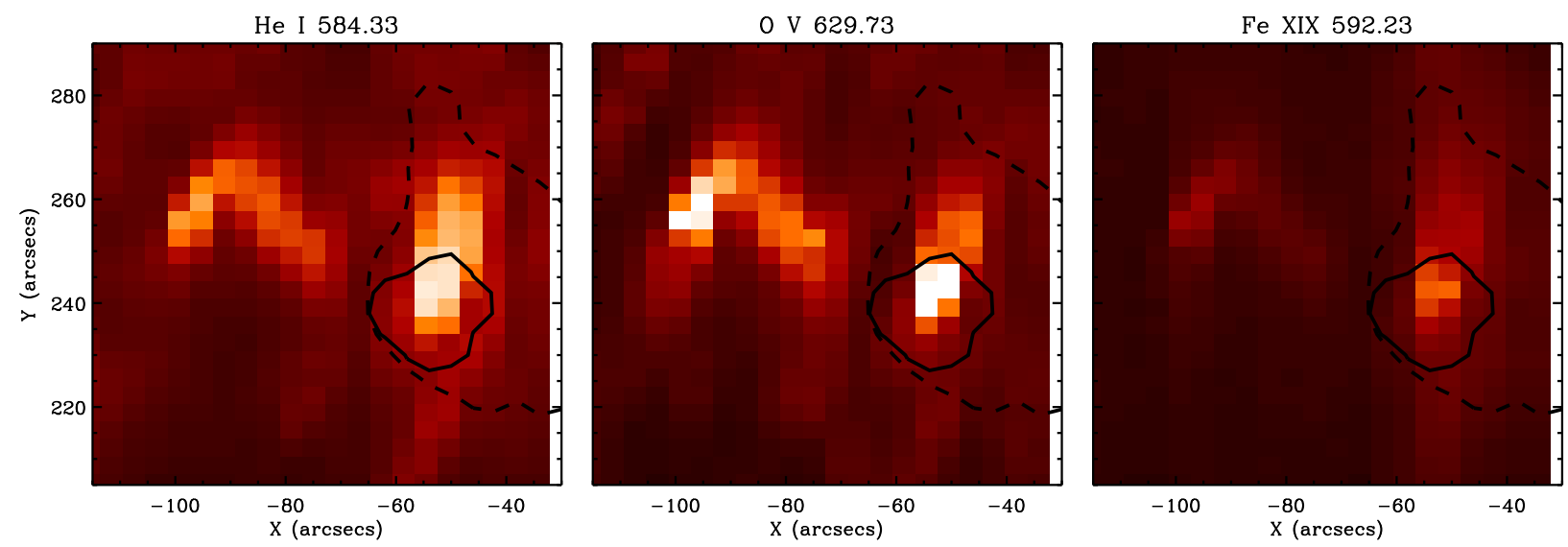

Fig. 1. - CDS images obtained in the He I $(\log \mathrm{T}=4.5), \mathrm{O} \mathrm{V}(\log \mathrm{T}=5.4)$, and Fe XIX $(\log \mathrm{T}=$ 6.9) emission lines observed during the impulsive phase of the 2002 July 15 solar flare. RHESSI 6-16 keV (dotted line) and 16-50 keV (solid lines) contours are overlaid, drawn at $5 \%$ and $10 \%$ of the peak intensity, respectively.

kilometers per second. This process is known as 'chromospheric condensation'. A strong case for explosive evaporation was presented by Brosius \& Phillips (2004), who reported oppositely directed flows using the Coronal Diagnostic Spectrometer (CDS; Harrison et al. 1995) on board the Solar and Heliospheric Observatory ( $\mathrm{SOHO}$ ) during a hard X-ray (HXR) burst. A more recent study by Milligan et al. (2006; hereafter referred to as Paper I) also found these flows patterns, but critically, were able to derive the properties of the driving electron beam using simultaneous HXR imaging and spectroscopy from the Reuven Ramaty HighEnergy Solar Spectroscopic Imager (RHESSI; Lin et al. 2002).

In this Letter we present the first observational evidence for gentle chromospheric evaporation due to nonthermal electrons during the impulsive phase of a solar flare. A brief overview of the instruments and data analysis is given in $\S 2$ (a more detailed description can be found in Paper I). Our results are then presented in $\S 3$, while a discussion and conclusions are given in $\S 4$.

\section{OBSERVATIONS}

Our study focuses on a compact GOES C9.1 flare, which began at 11:40:08 UT on 2002 July 15. The flare occured close to the solar meridian $\left(-40^{\prime \prime}, 232^{\prime \prime}\right)$ during a joint observing plan be- tween RHESSI and other ground- and space-based observatories. Unfortunately, there were no complimentary EUV images available from either the EUV Imaging Telescope (EIT) or the Transition Region and Coronal Explorer during the event.

The CDS observations reported here were aquired with the FLARE_AR observing sequence (see Paper I for details). Images taken during the impulsive phase in the He I (584.33 $\AA), \mathrm{O}$ V $(629.73 \AA)$, and Fe XIX (592.23 $\AA$ ) emission lines are shown in Figure 1. Spectra from each CDS pixel were fitted with a broadened Gaussian profile (Thompson 1999), for each of the spectral windows. Velocities were found by measuring Doppler shifts relative to quiet-Sun spectra, which were assumed to be emitted by stationary plasma. A heliographic correction was also applied assuming purely radial flows.

RHESSI is an imaging spectrometer capable of observing $\mathrm{X}$ - and $\gamma$-ray emission over a wide range of energies $(\sim 3 \mathrm{keV}-17 \mathrm{MeV})$. The thin attenuators on RHESSI were in place during part of the impulsive phase of this event, thus limiting the energy range to $\gtrsim 6 \mathrm{keV}$. Flare emission was not observed above $\sim 50 \mathrm{keV}$. The observing summary data for the energy ranges $6-12,12-25$, and $25-$ $50 \mathrm{keV}$, are shown in the top panel of Figure 2. As RHESSI went into eclipse at $\sim 11: 49: 00 \mathrm{UT}$, the GOES lightcurve for this event has been overplot- 

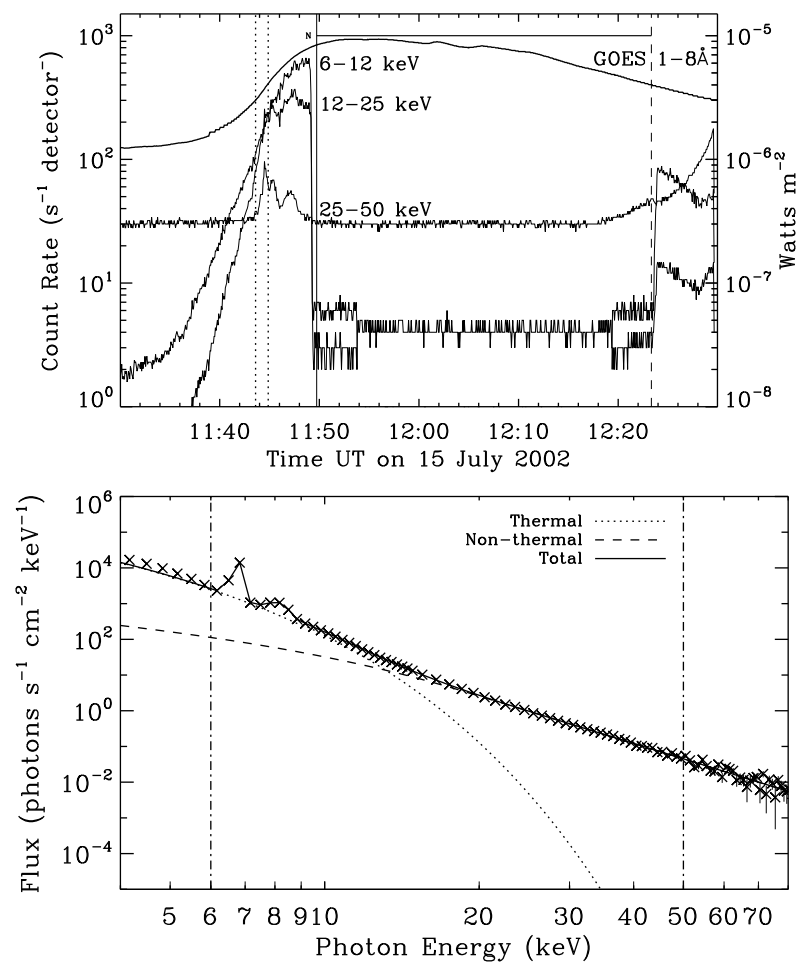

Fig. 2.- Top panel: RHESSI observing summary data (corrected for changes in attenuator states) from the $6-12,12-25$, and $25-50 \mathrm{keV}$ bands. Threm vertical dotted lines indicate the time interval over which images and spectra were obtained, corresponding to the observation of significant upflows with CDS. The bar denoted ' $\mathrm{N}$ ' at the top of the plot illustrates the period that RHESSI was in eclipse. Overplotted is the GOES 1-8 $\AA$ curve. Bottom panel: Portion of the RHESSI spectrum integrated over the time range given above. The energy range 6-50 keV lying between the vertical dot-dashed lines was fitted assuming an isothermal component (dotted curve) and a thick-target bremsstrahlung component (dashed curve).

ted for completeness. Both the RHESSI images and spectra were obtained over a 76 second period from 11:43:36-11:44:52 UT to coincide with the time range over which CDS observed blueshifts in the Fe XIX line. This time interval is indicated by two vertical dotted lines in the top panel of Figure 2 and includes the impulsive 25-50 keV HXR burst. RHESSI images in two energy bands (616 and $16-50 \mathrm{keV}$ ) were reconstructed using the
Pixon algorithm (Hurford et al. 2002). Contours at $5 \%$ and $10 \%$ of the peak intensity, respectively, in each band are overlayed on each EUV image in Figure 1. The RHESSI spectrum was fitted assuming an isothermal distribution at low energies and thick-target emission at higher energies (bottom panel of Figure 2).

\section{RESULTS}

The thick-target model solution consistant with the RHESSI photon spectrum produced an electron distribution with a low-energy cutoff $\left(\epsilon_{c}\right)$ of $\sim 20 \mathrm{keV}$, and a power-law index $(\delta)$ of $\sim 5.2$; a break energy of $\sim 20 \mathrm{keV}$ in the electron spectrum corresponds to a break energy of $\sim 16 \mathrm{keV}$ for the associated photon spectrum. From the properties of the inferred electron spectrum, the total power of nonthermal electrons was found to be $\sim 8 \times 10^{27} \mathrm{ergs} \mathrm{s}^{-1}$. The reconstructed $16-50 \mathrm{keV}$ image yielded an upper limit to the HXR source size of $\sim 1.8 \times 10^{18} \mathrm{~cm}^{2}$, and the resulting flux of nonthermal electrons was therefore found to be $\geq 5 \times 10^{9} \mathrm{ergs} \mathrm{cm}^{-2} \mathrm{~s}^{-1}$.

Figure 3 shows the spatial distribution of the plasma flows seen in each of the He I, O V, and Fe XIX lines. Net upflows of $13 \pm 16$ and $16 \pm 18 \mathrm{~km} \mathrm{~s}^{-1}$ were observed in the He I and $\mathrm{O} \mathrm{V}$ maps by averaging over all CDS pixels within the 16-50 keV $10 \%$ contour as observed by RHESSI. Moderately strong upflows of $110 \pm 58 \mathrm{~km} \mathrm{~s}^{-1}$ were observed in the Fe XIX map by averaging over the same area. Weaker flows of $\lesssim 50 \mathrm{~km} \mathrm{~s}^{-1}$ were measured away from this area and appear to be aligned with the thermal $(6-16 \mathrm{keV})$ emission observed by RHESSI. The mean velocity (from within the HXR emitting area) as a function of temperature for each of the five emission lines observed by CDS is shown in Figure 4. Error bars represent a $1 \sigma$ uncertainty. The values reported in Paper I for the case of explosive evaporation (electron flux $\geq 4 \times 10^{10} \mathrm{ergs} \mathrm{cm}^{-2} \mathrm{~s}^{-1}$ ) are overplotted.

\section{DISCUSSION AND CONCLUSIONS}

Simultaneous X-ray and EUV observations of gentle chromospheric evaporation during the impulsive phase of a C9.1 solar flare are presented using data from RHESSI and SOHO/CDS. Until now, studies that reported evidence for gentle evaporation were entirely focused on low-velocity 

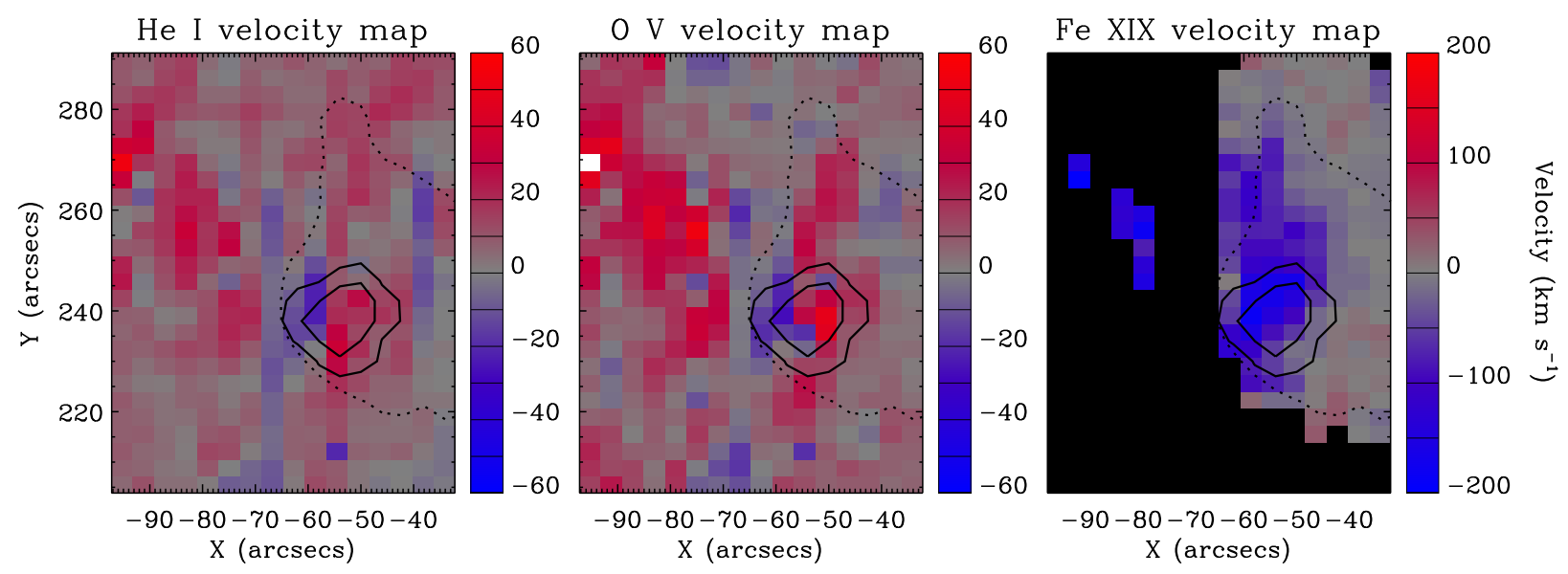

Fig. 3.- Velocity maps in the He I, O V, and Fe XIX lines. Downflows are indicated by red pixels, while upflows are given in blue pixels. The solid line denotes the RHESSI $16-50 \mathrm{keV}$ contours at $10 \%$ and $50 \%$ of the peak intensity, while the dotted contour shows the $6-16 \mathrm{keV}$ emission at $5 \%$ of the peak intensity.

$\left(\lesssim 80 \mathrm{~km} \mathrm{~s}^{-1}\right)$ mass motions observed during the decay phase of solar flares (Schmieder et al. 1987; Zarro \& Lemen 1988; Czaykowska et al. 2001; Berlicki et al. 2005). In each of these cases, the mechanism responsible was believed to be thermal conduction caused by the steep temperature gradients between the high-temperature flare plasma and the cool, underlying chromosphere (Antiochos \& Sturrock 1978). This was also motiviated by the fact that power-law spectra are not observed during the decay phase of the majority of solar flares.

In this Letter, we report the first observational evidence for gentle evaporation due to nonthermal electrons during the impulsive phase of a solar flare as predicted by current theoretical models. Upflows of $13 \pm 16,16 \pm 17$, and $110 \pm 58 \mathrm{~km} \mathrm{~s}^{-1}$ as seen in the He I, O V, and Fe XIX lines, respectively, result from a beam of non-thermal electrons with a flux value of $\geq 5 \times 10^{9} \mathrm{ergs} \mathrm{cm}^{-2} \mathrm{~s}^{-1}$. From Paper I, we reported that an order of magnitude higher flux $\left(\geq 4 \times 10^{10} \mathrm{ergs} \mathrm{cm}^{-2} \mathrm{~s}^{-1}\right)$ gives rise to downflows of $\sim 35$ and $\sim 45 \mathrm{~km} \mathrm{~s}^{-1}$ in the He I and $\mathrm{O}$ V lines, and upflows of $\sim 270 \mathrm{~km} \mathrm{~s}^{-1}$ in the Fe XIX line. As a consequence, the findings reported here and in Paper I support the theoretical models which predict that the response of the solar chromosphere is sensitively dependent on the flux of accelerated nonthermal electrons. Our results also support the prediction that there exists a threshold value for the nonthermal electron flux above which the chromosphere cannot efficiently radiate the deposited energy. Fisher, Canfield, \& McClymont (1985a) proposed that this threshold value is $\sim 3 \times 10^{10} \mathrm{ergs} \mathrm{cm}^{-2} \mathrm{~s}^{-1}$. Above this value, electron fluxes were shown to begin to drive chromospheric condensation, a process resulting from the high pressures reached by the rapidly expanding evaporated material. The combination of the results presented here and in Paper I also lend very strong evidence to support this principle.

The recent hydrodynamic simulations of Abbett \& Hawley (1999) and Allred et al. (2005) have been developed to include more realistic electron beam parameters and a non-LTE treatment of the solar atmosphere. These more detailed calculations still provide the distinction between gentle and explosive evaporation for differing nonthermal electron fluxes. These models will be further developed to include higher temperature plasmas that will be observed by the EUV Imaging Spectrometer (EIS) on board Solar-B, due for launch in late 2006. By combining EIS observations with RHESSI data in the future, an even greater understanding on the behaviour of high-temperature plasmas during solar flares will be achieved.

This work has been supported by a Department of Employment and Learning studentship in conjunction with a Cooperative Award in Science and Technology from NASA Goddard Space 


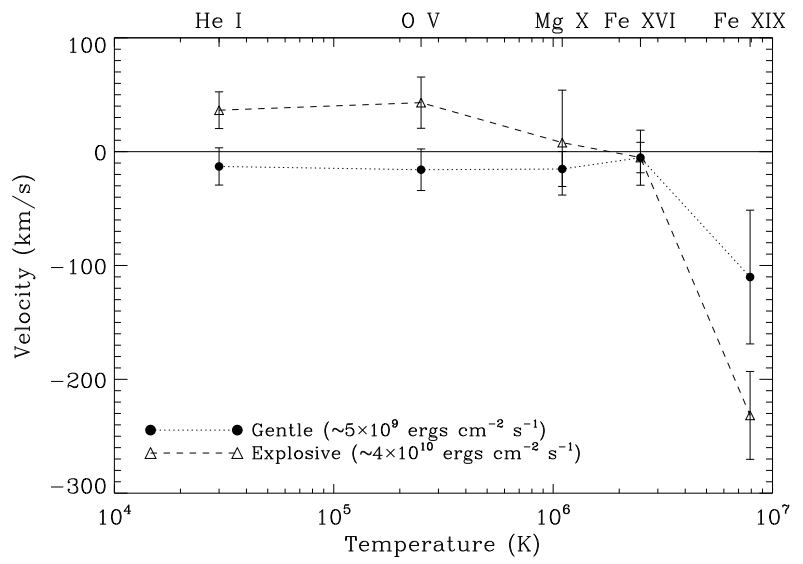

Fig. 4. - Plasma velocity as a function of temperature for each of the five lines observed using CDS. Positive velocities indicate downflows, while negative values indicate upflows. The data points plotted with solid circles denote the values presented in this study, while the triangles illustrate those values presented in Paper I for the case of explosive evaporation. The dotted and dashed lines connecting the points are added to guide the reader.

Flight Center. F. P. K. is grateful to A. W. E. Aldermaston for the award of a William Penny Fellowship. We would like to thank Brian Dennis and the RHESSI team, Joe Gurman and Dominic Zarro at Goddard for their continued support. $\mathrm{SOHO}$ is project of international collaboration between the European Space Agency and NASA.

\section{REFERENCES}

Abbett, W. P., \& Hawley, S. L. 1999, ApJ, 521, 906

Allred, J. C., Hawley, S. L., Abbett, W. P., \& Carlsson, M. 2005, ApJ, 630, 573

Antiochos, S. K., \& Sturrock, P. A. 1978, ApJ, 220,1137

Berlicki, A., Heinzel, P., Schmieder, B., Mein, P., \& Mein, N. 2005, A\&A, 430, 679

Brosius, J. W., \& Phillips, K. J. H. 2004, ApJ, 613,580

Brown, J. C. 1971, Sol. Phys., 18, 489
Czaykowska, A., Alexander, D., \& De Pontieu, B. 2001, ApJ, 552, 849

Fisher, G. H., Canfield, R. C., \& McClymont, A. N. $1985 a$, ApJ, 289, 414

Fisher, G. H., Canfield, R. C., \& McClymont, A. N. 1985b, ApJ, 289, 425

Fisher, G. H., Canfield, R. C., \& McClymont, A. N. $1985 c$, ApJ, 289, 434

Harrison, R. A., et al. 1995, Sol. Phys., 162, 233

Hurford, G. J., et al. 2002, Sol. Phys., 210, 61

Lin, R. P., et al. 2002, Sol. Phys., 210, 3

Milligan, R. O., Gallagher. P. T., Mathioudakis, M., Bloomfield, D. S., Keenan, F. P., \& Schwartz, R. A. 2006, ApJ, 638, L117

Schmider, B., Forbes, T. G., Malherbe, J. M., \& Machado, M. E. ApJ, 1987, 317, 956

Thompson, W. T. 1999, CDS Software Note No. 53

Zarro, D. M., \& Lemen, J. R. 1988, ApJ, 329, 456

This 2-column preprint was prepared with the AAS LATEX macros v5.2. 